\title{
Knife-Edge Scanning Microscopy for Bright-Field Multi-Cubic Centimeter Analysis of Microvasculature
}

\author{
Michael J. Pesavento, Caroline Miller*, Katy Pelton, Molly Maloof, Corey E. Monteith, \\ Venkata Vemuri, and Megan Klimen \\ 3Scan, Inc., 2122 Bryant St., San Francisco, CA 94110 \\ *mills@3scan.com
}

\begin{abstract}
Serial section microscopy has proven to be a powerful tool in examining the 3-dimensional (3D) nature of tissue; however, it is limited by the size of tissue samples that can be used and the manual process of acquiring data. The knife-edge scanning microscope (KESM) acquisition and analysis computational system resolves these issues by providing high-resolution light microscopy image data at multi-cubic centimeter scale. Using this system for quantification of microvasculature provides the ability to examine, measure, and compare the 3D nature of vascular networks at whole organ scale and sub-micron resolution.
\end{abstract}

\section{Introduction}

Modern light microscopy has benefited from the use of serial section microscopy such as in the Allen Brain Atlas and the creation of new rodent models for Alzheimer's disease research $[1,2]$. Typically, slides are prepared in the conventional manner, where images are collected using traditional light microscopy and digitally scanned. The resulting images then must be computationally registered to one another to create a 3D image. However, numerous issues have inhibited widespread adoption of this method. These include the manual nature of slide preparation and staining, the introduction of artifacts from manual sectioning and mounting on slides, the time taken for whole-slide scanning an entire sample, the difficult nature of image registration and data handling, and the simple fact that it results in too much data for modern infrastructures to handle.

The knife-edge scanning microscope (KESM) combines sectioning and imaging into a single step, automates a large part of the traditional pathology workflow, and allows for a greater speed, precision, throughput, and scale at which tissues are digitized (Figure 1). The KESM achieves tissue data collection at a resolution of submicron pixels with a maximum sample volume of over $100 \mathrm{~cm}^{3}$, exceeding the depth offered by confocal or 2-photon microscopy. To handle high-resolution data of over a terabyte per $\mathrm{cm}^{3}$, sophisticated data processing software is applied to model $3 \mathrm{D}$ tissue reconstructions, provide interactive image views, and apply quantitative analytics. This allows quantitative analysis to be performed on 3D image stacks of whole mouse organs, which is extremely difficult, expensive, and time-consuming with traditional manual techniques.

The KESM was first developed at the Brain Network Laboratory at Texas $\mathrm{A} \& \mathrm{M}$ as a neuroscience tool to examine brain microvasculature and neuronal microcircuitry in 3D [3]. The KESM technology provides extremely high-throughput processing, sectioning, and 3D image generation of samples. This has attracted significant interest from the research community, the drug development community, and academic pathologists who are looking for new methods to bring histopathology into the digital age. Multiple imaging modalities of KESM (mono- and tri-chromatic bright-field plus multi-channel fluorescence) are in development to bring this technology to a broader range of scientists, including clinical pathologists. This article explains the design of the microscope, tissue preparation, how the KESM works, software reconstruction, some examples of data outputs, an application to mouse brain tissue, and the significance of this novel imaging technique.

\section{Materials and Methods}

Preparation of the tissue block. Mouse clearing, fixation, and India ink perfusion are performed as follows. The mouse is anesthetized using isoflurane and tested for toe pinch response prior to the beginning of the non-survivable surgical technique. A blunted 18-gauge needle is placed into the left ventricle of the heart, and the right atria is snipped to allow return flow. Mice are perfused at a pressure of $300 \mathrm{mmHg}$ using a custom pressurized perfusion rig (Figure 2). We have found that perfusing at high pressure ensures more complete staining of the microvasculature compared to lower-pressure perfusion techniques.

The mouse is first perfused with phosphate buffered saline (PBS) until the blood runs clear. Following clearing, the mice are perfused with $200 \mathrm{~mL}$ formalin; this long formalin perfusion provides excellent fixation of the tissues and allows time for the vasculature to fix in an "open" position. This ensures complete

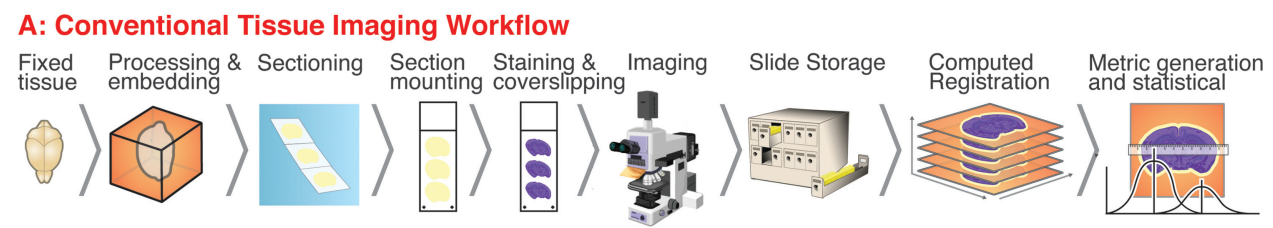

B: KESM Tissue Imaging Workflow

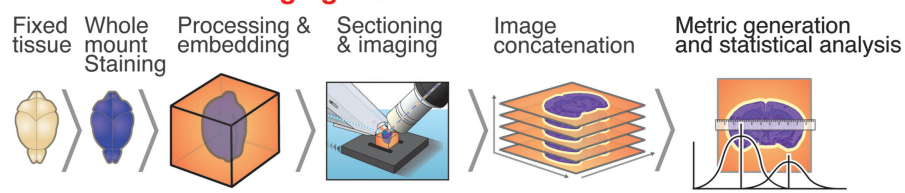

Figure 1: Rethinking imaging workflow. (A) Current tissue imaging pathway, where tissue is embedded, sectioned, stained, and imaged, and each image must then be registered to one another. (B) KESM automation of this process to produce $3 \mathrm{D}$ stacks of images that only require concatenation. 


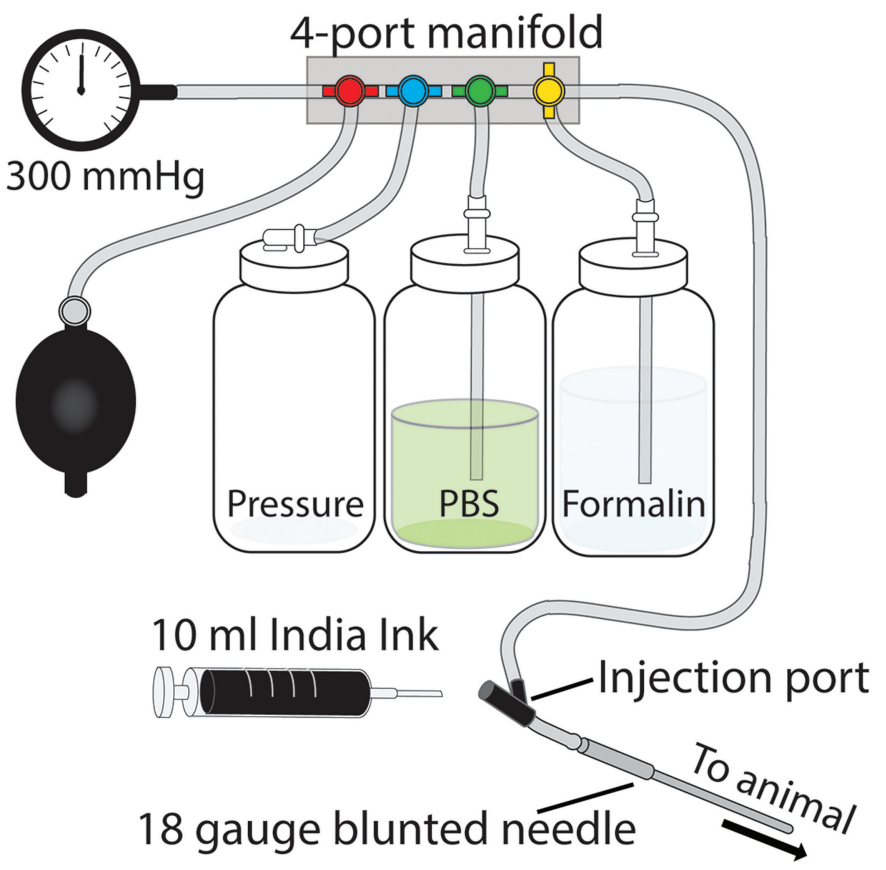

Figure 2: Perfusion rig. A homemade pressure system was used to perfuse the mice with a colloidal solution of carbon black, which renders their vasculature as black on a white tissue background. The whole system is pressurized to $300 \mathrm{mmHg}$. Mice are cleared with phosphate buffered saline (PBS) and fixed with formalin; then the injection port is used to inject $10 \mathrm{ml}$ of India ink into the vascular system.

filling of the vessels and capillary beds with the $10 \mathrm{~mL}$ colloidal solution of carbon (India ink) that follows. Organs of interest are dissected from the animal and left in formalin to further fix overnight. The following day tissue samples are processed and embedded in LR white resin; briefly, the tissues are dehydrated to $100 \%$ ethanol and then subjected to increasing concentrations of LR white to $100 \%$ resin. Tissue and resin are then polymerized in a $65^{\circ} \mathrm{C}$ oven for 24 hours. The resulting block of resin and tissue is secured onto a custom sled for our microscope.

Sample imaging. The KESM was designed to combine a microtome and a microscope into a single tool. The KESM comprises three major components (Figure 3A): (1) the knife arm assembly, (2) the optics train and camera, and (3) the stage and water bath. The knife arm assembly and optics train remain stationary during imaging. The key feature of the KESM is the diamond knife arm assembly (Figure $3 \mathrm{~B}$ ), which functions as both a sectioning tool and the illumination source for the microscope. The removable diamond knife is mounted on a fixed knife arm with a channel running above and below. The channel on the underside of the knife arm contains a fiber optic cable that connects the LED light source to the back of the diamond knife, providing illumination that is transmitted through the tissue section to the line-scanning camera. The channel above the knife arm creates a closed-loop water channel that pulls the tissue section away from the objective lens as the tissue is being sliced and directs the sectioned piece into a filter to be discarded.

Perpendicular to the knife arm assembly is the optics train with a $10 \times$ water-immersion objective (Zeiss $10 \times / 0.3 \mathrm{NA}$ achromat) focused on the bevel of the diamond knife. A high-speed line-scan time-delay integration (TDI) camera (Dalsa Teledyne Piranha HS80-08k80) is coupled with the objective lens to capture line-scanned images of the tissue as it moves over the bevel of the knife. The tissue is mounted inside the water bath and is moved across the bevel of the stationary knife by the motion of the high-precision mechanical air-bearing Aerotech stage $(X Y$ precision $= \pm 250 \mathrm{~nm} ; Z$ precision $= \pm 300 \mathrm{~nm})$. The stage moves along the $X$ (vertical) axis to cut $2 \mathrm{~mm}$-wide sections of tissue as thin as $0.5 \mu \mathrm{m}$. For tissue blocks wider than $2 \mathrm{~mm}$, the KESM moves along the $Y$ axis to take slices that are later stitched together using software to create a full face. After each face has been sectioned, the $Z$ stage moves the tissue up at a fixed slice thickness to start sectioning the next face.

KESM control. An operator controls the KESM by logging into a web interface in a browser window on a laptop or tablet (Figure 4). The user interface allows the operator to set the physical cutting space in three dimensions (XYZ) (panel 1), depending on the size of the tissue block, and also allows the

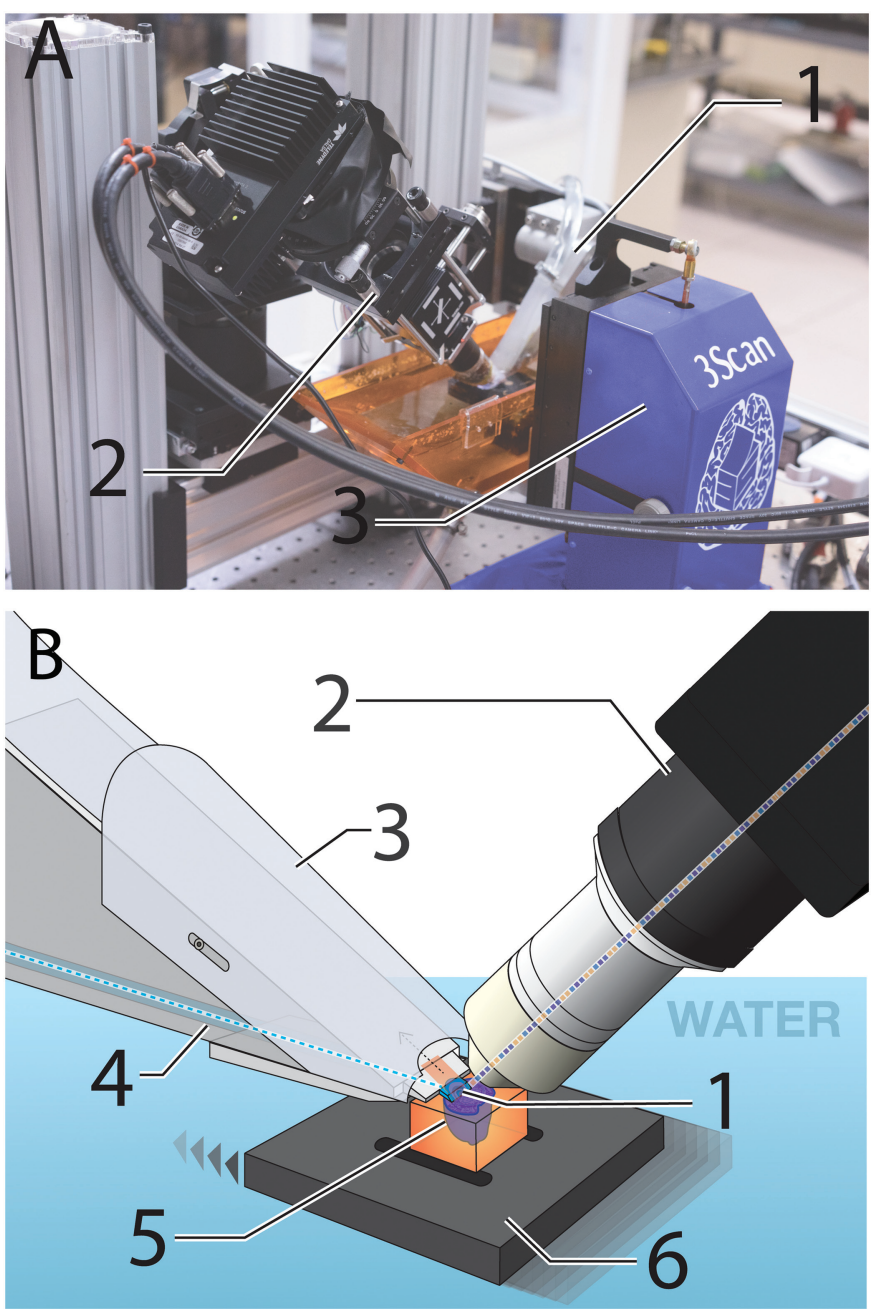

Figure 3: Overview of KESM mechanical components. (A) The knife-edge scanning microscope consists of (1) the knife arm assembly, (2) the optics train and sensor, and (3) the water bath and stages. (B) Schematic view of the (1) diamond knife, (2) the objective lens, (3) suction channel, (4) fiber optic cable, (5) embedded tissue, and (6) precision $X Y Z$ stage. 


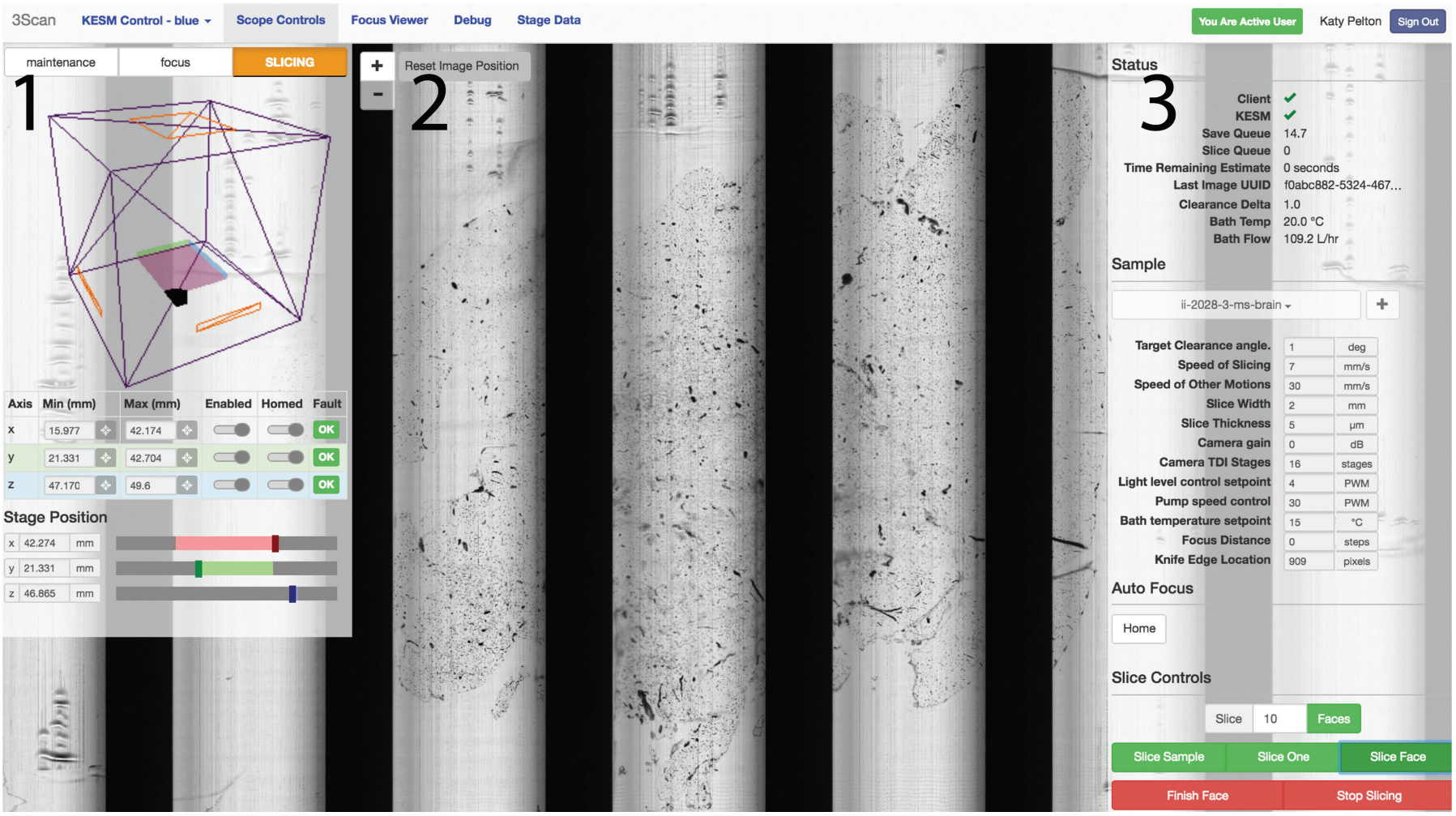

Figure 4: KESM control screen. The operator controls the KESM through a browser-based interface. This interface allows operators to (1) set sample volume cutting parameters, (2) view the most recent images, and (3) vary cutting parameters, such as speed of slicing and slice thickness, to improve image quality. This view shows raw data as acquired including the knife edge, and non-tissue areas of the camera sensor, which are seen as black stripes. During image processing these are removed, and the resulting images are stitched together.
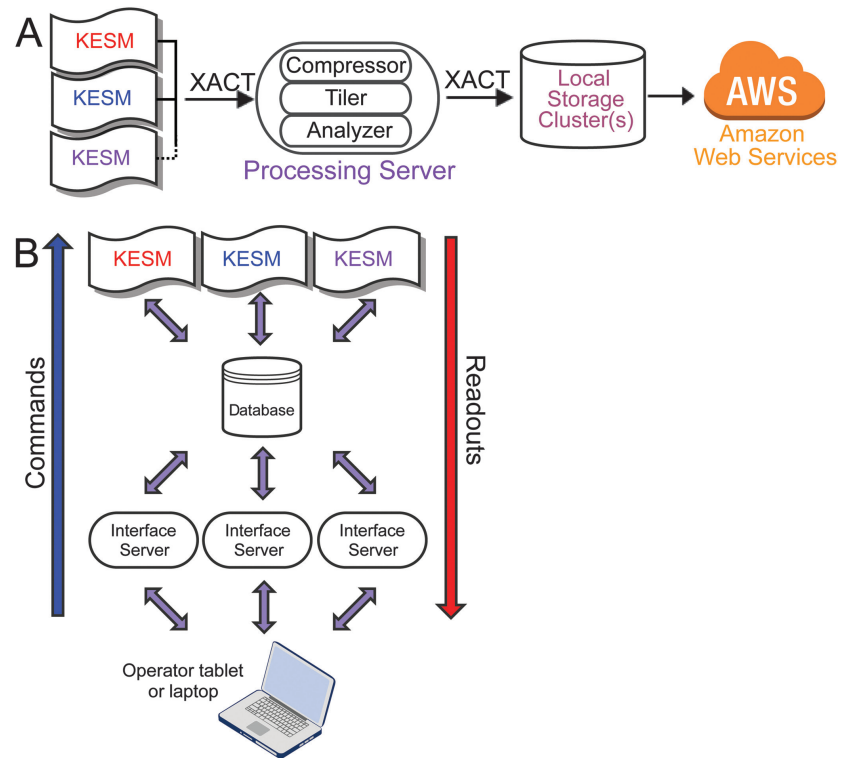

Figure 5: Data flow in the KESM. (A) Data are received by the process, which retains the raw image data in RAM until all processing is completed. It shares this memory with the three: the Image Compressor, Image Tiler, and Image Analyzer. Image Compressor compresses the image to a PNG bytestream in memory and then sends the compressed image to the local storage cluster. Image Tiler generates a tile pyramid from the raw image. Image Analyzer generates basic image statistics from the raw data and inserts the statistics in a queryable database. These images are sent to the AWS S3 cloud. (B) Database reads commands from KESM operator to enable cluster synchronization and can control multiple KESMs on a web browser from any electronic device. operator to set slicing parameters, such as slicing speed $(\mathrm{mm} / \mathrm{s})$, slice thickness $(\mu \mathrm{m})$, clearance angle, water bath temperature, light level, and other parameters (panel 3). A major feature of the system is that the user interface displays the most recently acquired images in a tile viewer within tens of seconds after slicing (panel 2). The operator can also see the status of the microscope in real time on any device with an internet browser, including mobile devices and tablets. This quick visual feedback allows the operators to adjust the slicing and imaging parameters to optimize the imaging and react promptly to any issues that might arise.

Image acquisition and storage. As the KESM takes a slice and the camera records the data, the pixel data of each image go through multiple processing steps using a scalable framework across a cluster of image processing computers. The KESM sends the image data to any machine in the cluster using 3Scan's XACT communication protocol, a framework that facilitates transactional exchanges for high-throughput and high-fidelity communication among multiple computers (Figure 5A). Data are acquired by the KESM and passed to the imaging processing cluster via XACT, where the Receptionist process shares raw image data with three daughter processes: data are analyzed to generate basic image statistics, multiresolution image tiles (pyramidal tiling) are produced, and the images are compressed in PNG format-all in parallel. After these processes are complete, image data are passed to local storage via the XACT protocol. A local copy is maintained for approximately two months to facilitate low-latency image 
viewing and processing. Eventually the entire dataset is sent to Amazon Web Services (AWS) (Figure 5A).

Database operations. The KESM software operates the local processing and storage clusters in a decentralized manner to prevent single points of failure and to facilitate incremental improvement. The database (Figure 5B) serves several critical functions: (a) storing image metadata, (b) relaying commands to the KESM from the user interface or from programmatic manipulation of the database, (c) synchronizing computational processing via interlock checks for completed tasks, and (d) providing data for the user interface servers.

Image analysis. Once the slice images from the microscope are stored, they must be stitched together to create a large $3 \mathrm{D}$ volume representing the digitized tissue. Fidelity of the reconstruction requires multiple image processing steps such as cleaning and filtering, as well as later steps that compress the data into object classification and object metrics. The scale of
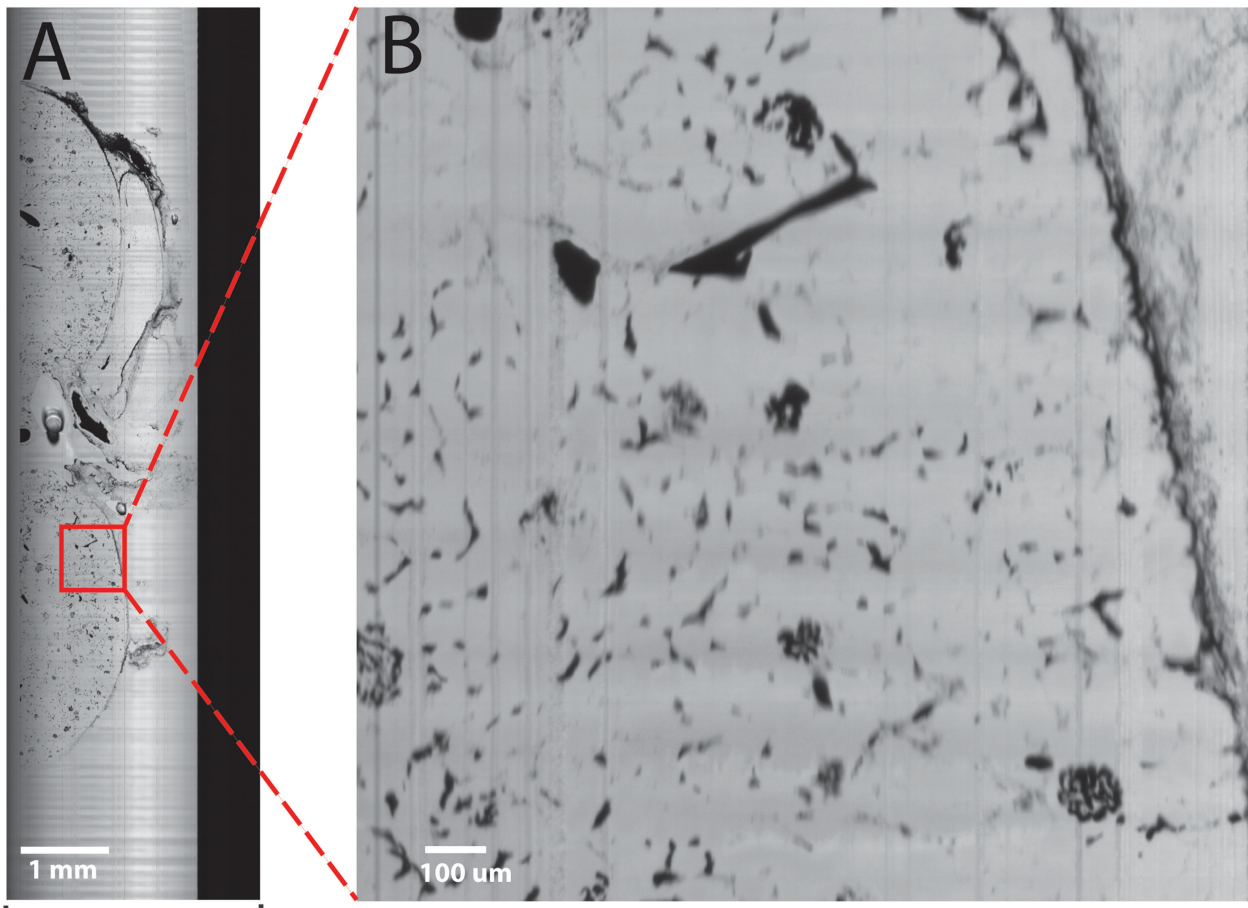

4096 pixels

Figure 6: Cropped image slice and full-resolution zoom of mouse kidney with India ink localized vasculature. Raw images are shown as acquired from the KESM; no post-processing was done. The larger image shows a cropped slice of $12,500 \times 4,096$ pixels or 50 megapixels. The usable portion of the slice is restricted to the field of view of the objective, or about $2 \mathrm{~mm}$ wide for the objective lenses used and is 2,858 pixels wide per slice. The black stripe is the un-illuminated edge of the knife. Original resolution for both images was $0.7 \times 0.7 \mu \mathrm{m} / \mathrm{pixel}$. data compression spans from trillions of bytes down to a single byte that can represent a single measurement from an entire organ or other piece of tissue.

The sample slices are stored as narrow strips 4,096 pixels wide (Figure 6). The physical limits of the stage restrict our sample size to $50 \times 50 \times 20 \mathrm{~mm}$. This translates to 70,000 pixels across or 25 slices wide. With an average slice thickness of $5 \mu \mathrm{m}$, the KESM can take up to 4,000 slices across the $Z$ depth. This translates to a maximum volume of 70,000 $\times 70,000 \times$ 4,000 pixels or 19.6 teravoxels (Tv) of data. After initial digital isolation of the tissue of interest, such as the mouse brain examined in this article, the region of interest might be 20,000 $\times 15,000 \times 1,000$ pixels (approximately $14 \times 10.5 \times 5 \mathrm{~mm}$ ) or 300 gigavoxels $(\mathrm{Gv})$.

A distributed processing framework is necessary to transform imaging data at this scale. The Apache Spark platform uses a MapReduce framework, which makes it easy to rapidly process terabytes of data across as many CPUs as needed. Amazon's Elastic Computing Cloud (EC2) allows adaptive scaling of computational infrastructure to match needed processing requirements. Amazon's Simple Storage Service (S3) serves as our storage solution for all analysis outputs, as well as the original raw slice images. Use of distributed storage and distributed processing with S3 and EC2, respectively, dramatically reduces the time to write and read the images from hard disk, one of the largest time bottlenecks in processing data of this magnitude. The Python 3 programming language is used for all analysis and leverages many available libraries, such as numpy, scipy, and openCV.
In the first stage of image processing, a cuboid region of interest (ROI) containing the tissue to be examined is identified. Once the ROI has been selected, the raw slices are stitched together (Figure 7A). Due to the high precision of the KESM stage motion, no image registration transformations are necessary to align the tissue features; voxels in the digital volume are aligned in correspondence with how the tissue is sectioned from the block. Therefore, slice image arrays can be cropped and concatenated to create a 2D face (Figure 7B).

Once stitched, standard algorithms are used to clean mechanical and optical artifacts (Figure 7C). In the example presented here, blood vessels stained with India ink are the objects of interest and are segmented with one of many available segmentation algorithms [4-7] (Figure 7D). Segmented vessels are then thinned to their skeleton or the topological equivalent as a single pixel traces through all branches [8-10]. This skeleton is then vectorized in a graph, and measurements are taken from this representation. Metrics are calculated from classified vessel segments and branch points [11] (Figure 7E). Finally, the 3D voxel data can be combined to create a $3 \mathrm{D}$ rendering of the tissue that allows views of entire organs (Figure 7F).

\section{Results}

To compare the vascular networks from different areas of a mouse brain (Figure $8 \mathrm{~A}), 360 \mu \mathrm{m}$ cubes $(512 \times 512)$ pixels per face, $5 \mu \mathrm{m}$ slice thickness) from the forebrain neocortex (somatomotor area) and cerebellum were digitally isolated (Figure 8B). Volumetric and vectorized measurements were 

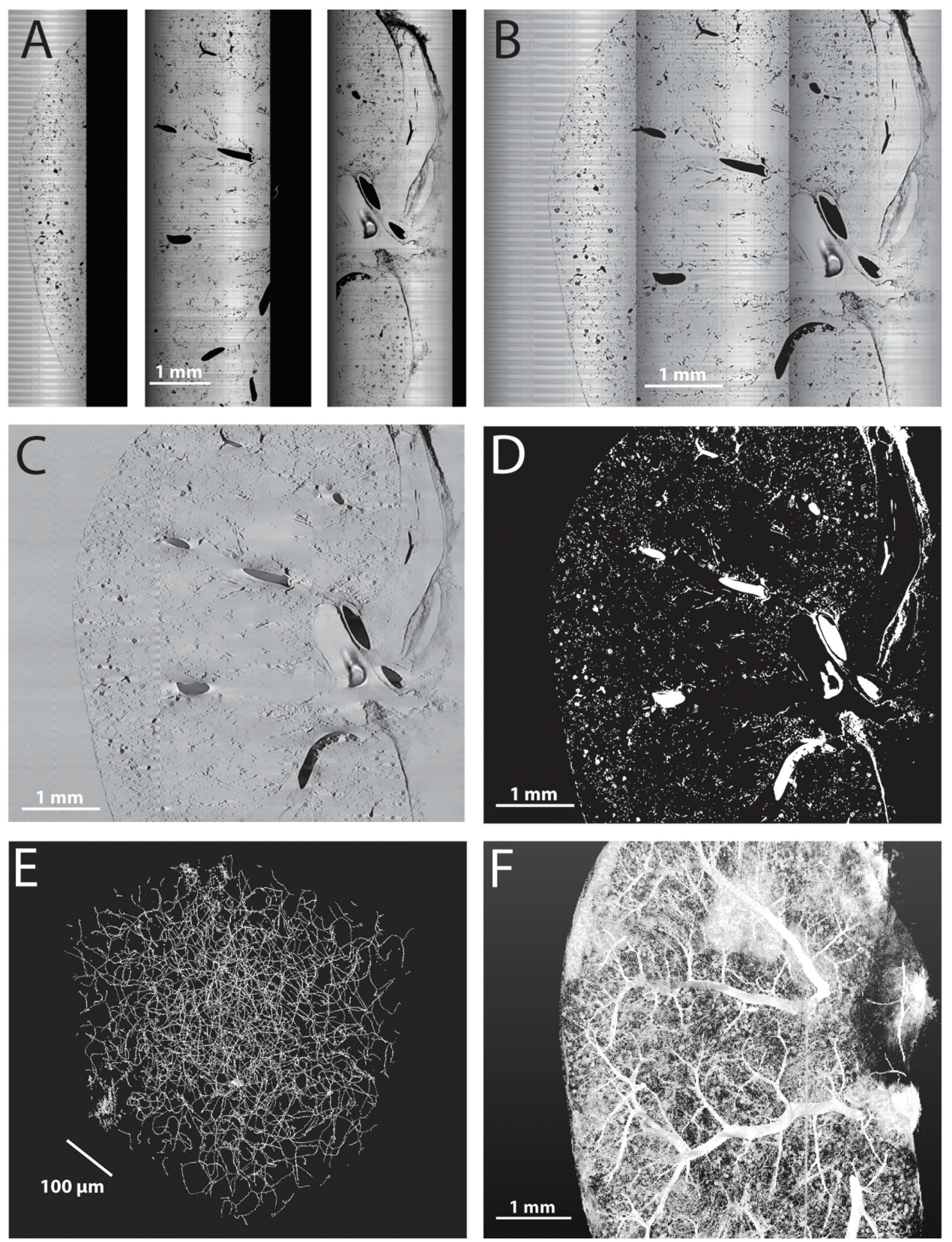

Figure 7: Image processing steps for grayscale KESM data. (A) First state of acquired image data saved as slices with metadata containing position coordinates as well as camera capture data. (B) An ROI is selected within a bounding cuboid. (C) Known mechanical artifacts in KESM are removed, such as uneven illumination or mechanical limitations in cutting physics ("chatter"). (D) Object classification is performed via 3D object segmentation. Here, blood vessels are identified based on intensity, distance, and morphological criteria. (E) Raster images are transformed into a graph-based representation of the vascular network. (F) The 2D raster images are stacked to create a 3D rendering of the tissue, with typical ROls in the hundreds of gigabytes range.
Typical microvascular studies examine the number of vessels in a $2 \mathrm{D}$ image that corresponds to the viewable area of the slice obtained through traditional histological techniques. Direct comparison to vascular metrics in other studies is challenging because of the lack of standardization of these metrics in the field, both for 2D and volumetric analysis $[12,13]$. However, a few measurements are commonly used across studies of microvasculature. Here we examine vessel density per unit volume, the ratio between vessel surface area and volume, and vessel segment tortuosity. These metrics provide a comparison to prior vascular studies in both $2 \mathrm{D}$ and $3 \mathrm{D}$. Because of the non-Gaussian distributions of both volumetric and vectorized measurements, a non-parametric Wilcoxon rank-sum test was used to compare significant differences between distributions in the two regions.

We compare vascular density (number of vessels $/ \mathrm{mm}^{2}$ ) between 2D faces of the forebrain and cerebellum volumes, aligned across the $Z$ axis of the volume (Figure 9A). Each comparison along the $Z$ axis is equivalent to the vessel density examined in single images in other studies [13]. Note how the density changes depending on the depth of the 2D face images, indicating the potential bias in the reduced sampling of $2 \mathrm{D}$ faces typically performed with other microscopy methods.

The mean vascular density per face was found to be significantly higher in the cerebellum than the forebrain (Wilcoxon rank-sum, $Z=-9.40, \mathrm{p}<1 \mathrm{e}-21$ ) (Figure 9C). A high degree of variability in vascular density between $2 \mathrm{D}$ faces for both regions was discovered, with a range compared for each cubic volume (Figure 8C, 8D). Volumes from a mouse brain perfused with India ink were sliced sagittally with a $5 \mu \mathrm{m}$ thickness in the $Z$ direction, giving a voxel size of $0.7 \times 0.7 \times 5 \mu \mathrm{m} /$ voxel. Slices were taken sagittally from the medial to lateral aspect. Each cubic region $(512 \times 512 \times 72$ voxels) represents $0.046 \mathrm{~mm}^{3}$ of brain tissue. The fractional volume of observed microvasculature (vascular voxel count/ total voxel count) in the forebrain was $1.07 \%$, compared with $3.18 \%$ in the cerebellum. spanning from 150 to 200 vessels per $\mathrm{mm}^{2}$ over a $Z$ depth of $360 \mu \mathrm{m}$ (72 slices), indicating the importance of identification and verification of structures across all three dimensions of tissue analysis (compare to Figure 9A).

Another important metric in microvasculature is the surface area to volume ratio, which directly affects the diffusion of oxygen and other molecules into the surrounding tissue. The forebrain region shows a significantly higher surface area to volume ratio (Wilcoxon rank-sum, $Z=9.81, \mathrm{p}<1 \mathrm{e}-21$ ) 
(Figure 9B). When distributions of volume and surface area are compared directly, the forebrain region shows a significantly lower volume $(Z=-10.24$, $\mathrm{p}<1 \mathrm{e}-23)$ and significantly lower surface area $(Z=-10.10, \mathrm{p}<1 \mathrm{e}-23)$ than the cerebellum. In the regions measured, the cerebellum had a large arteriole, which may account for much of the observed difference in volume and surface area.

From the vascular network graphs generated from skeletonized volumes (red lines inside volume renderings of Figure 8C, 8D), vessel segments are identified as the list of nodes between a branch point (bifurcation point of the graph) and an end point (graph terminus), two branch points, or two end points.

A commonly cited metric for microvasculature is the tortuosity of each vessel, measured as the Euclidean distance between the segment start and end points divided by the full path length of the segment [12]. A value of 1 indicates that the vessel length is the same as its distance, that is, the path is a straight line between two points. Values much greater than 1 indicate a higher degree of tortuosity, or deviation from the straight line approximation. In the samples examined, the cerebellum had a significantly higher tortuosity than the forebrain (Wilcoxon rank-sum test, $Z=9.37, \mathrm{p}<7 \mathrm{e}-21$ ) (Figure 9d). Future advancements in vessel classification algorithms will increase the accuracy and precision of measurements of the microvascular network.

\section{Discussion}

The KESM brings together elements of machine vision, automated serial sectioning, micromachining, advanced optics, and robotics to produce 3D images that can be used to quantify, manipulate, and analyze tissue structures. The following paragraphs review some of the advantages of this new method.

Imaging scale and resolution. The KESM fills a need for high-resolution imaging at high volume. With the stage limits listed in the Methods section above, this process enables a maximum sample volume of over $100 \mathrm{~cm}^{3}$ at sub-micron resolution, providing the capacity to image whole murine organs and large sections of higher animal organs. Because up to a terabyte of data is produced per $\mathrm{cm}^{3}$ tissue, accompanying
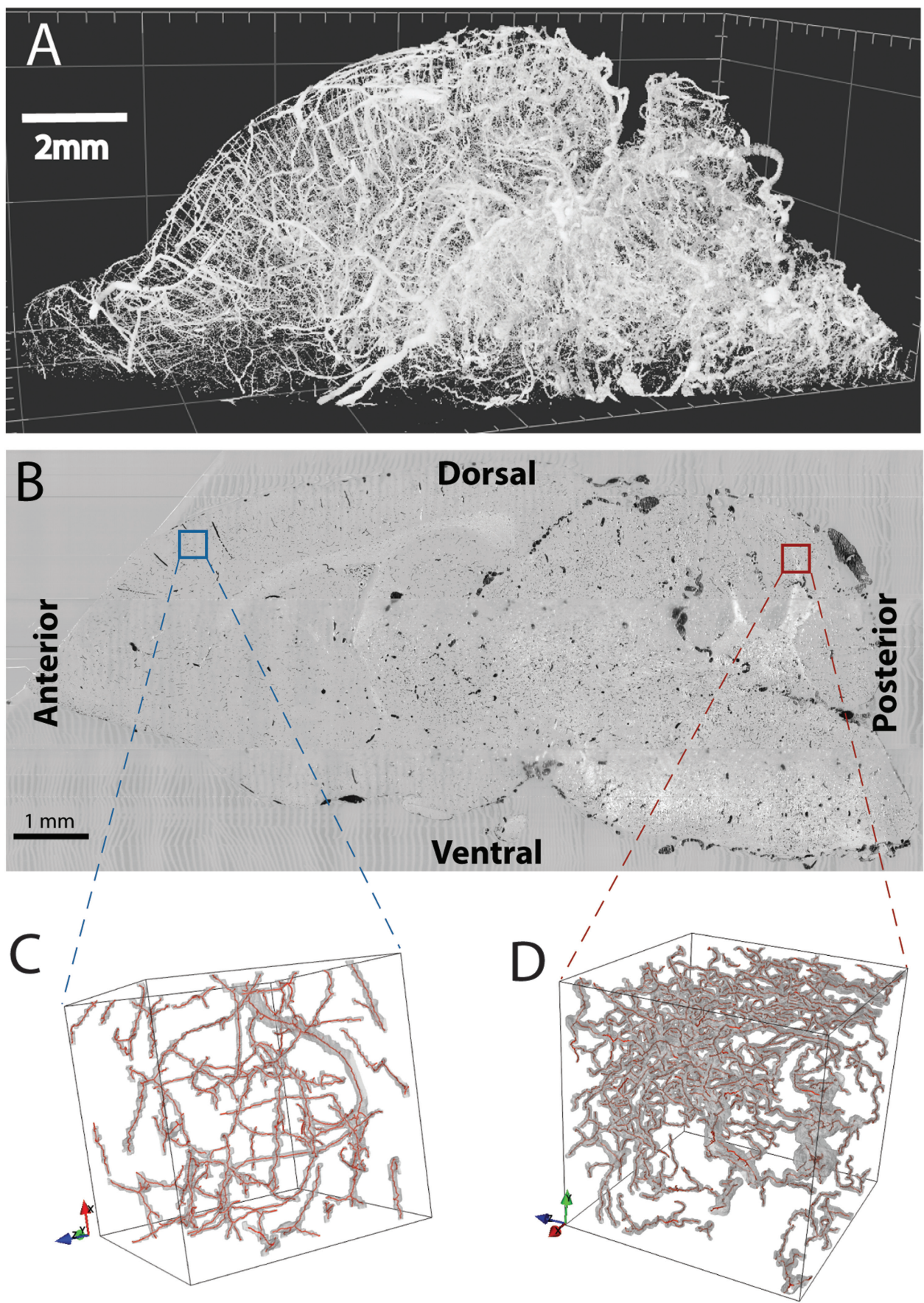

Figure 8: Selection of volumes of interest in whole mouse brain vasculature. (A) 3D rendering of microvasculature from mouse brain left hemisphere. Voxels were downsampled by $16 \times$ to create the render; only prominent vessels are shown. (B) Sagittal section of mouse brain, after stitching and cleaning. Boxes indicate the location and size of the subregions sampled: blue = forebrain, red = cerebellum. $(C, D)$ Renderings of the microvasculature (gray) and skeleton (red) of the selected subregions, where $(C)$ is from the forebrain and (D) is from the cerebellum.

software facilitates the processing of large swaths of data, allowing quantitative analytics and a wide variety of interactive viewing methods.

Image registration. Because of the precise stage motion in the KESM, tissue sectioned from the block can be concatenated into $3 \mathrm{D}$ images through alignment of the voxels in the digital volume. This removes the impediment of computationally expensive and imperfect image registration 

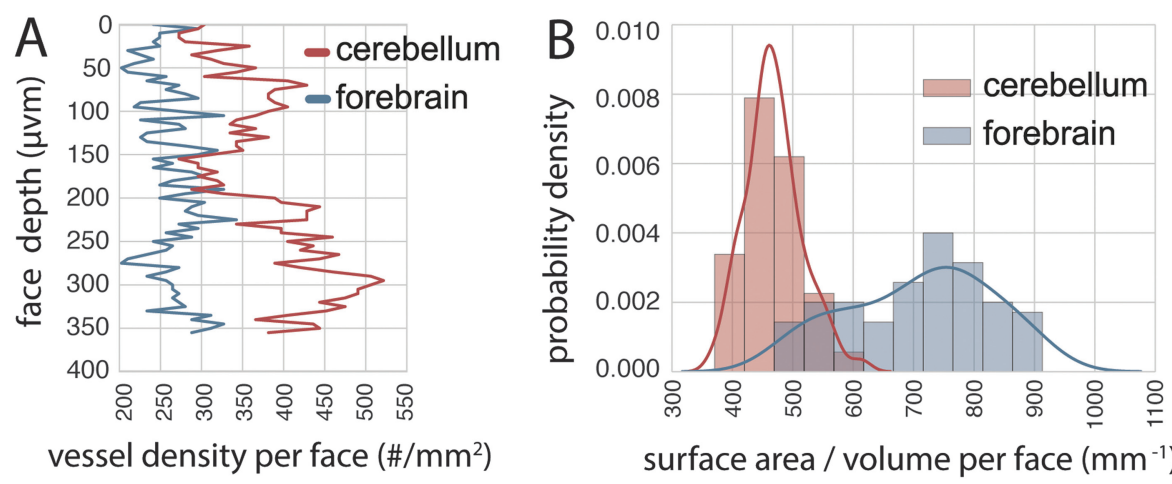

surface area / volume per face $\left(\mathrm{mm}^{-1}\right)$
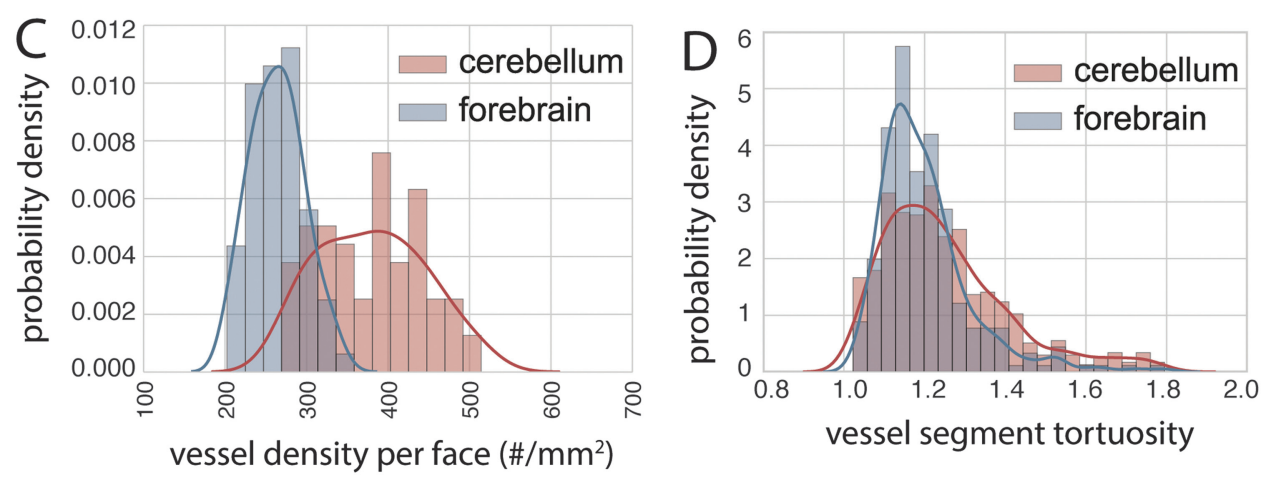

Figure 9: Comparison of volume-based metrics of microvascular networks in the forebrain cortex and cerebellum of a mouse. Cerebellum is shown in red, forebrain neocortex shown in blue. (A) Z-depth comparison of vessel density (number of vessels per $\mathrm{mm}^{2}$ ); each face depth value is equivalent to examining the density for a single $2 \mathrm{D}$ image. (B) Distribution of surface-area-to-volume ratio over all faces in the sample volume. (C) Distribution of vessel density over all 2D faces in the sample volumes, obtained by flattening the $2 \mathrm{D}$ values from $A$ into a histogram. (D) Distribution of segment tortuosity from vectorized vessel traces.

\section{Conclusion}

Serial section microscopy is difficult to achieve on organ scale because of the manual nature of sectioning, scanning, and reconstructing images from $2 \mathrm{D}$ slides. The KESM is the centerpiece of a $3 \mathrm{D}$ pathology platform designed to solve problems currently inhibiting the widespread application of serial section microscopy by automating the process of 3D tissue imaging. The KESM data can be used by researchers to quickly gather and analyze high-precision 3D images. Quantifying biological differences within an organ, or between organisms, is especially useful for animal models used in pre-clinical drug discovery research [14]. This enables quantification of microvasculature and may provide insight into various diseases, such as Alzheimer's, diabetes, and cancer. By examining large volumes of tissue at maximum light microscopy resolution with high throughput, scientists and pathologists can improve the accuracy of morphological assessment and identification of normal and diseased tissue states. transformations required to reconstruct traditional wholeslide images into $3 \mathrm{D}$ volumetric views.

Workflow efficiency. By slicing and scanning in one step, the KESM automated imaging system is a redesign of the microscopy workflow that provides many advantages over traditional microtome and imaging methods. In addition to replacing manual glass slide creation and storage, it reduces the time needed to collect large volumes of data at submicron resolution. Downstream processing of data is more efficient because the images need minimal registration to create a volume image.

Tissue quantification. The KESM significantly improves the quantification of microvasculature imaging in comparison to estimations made from 2D images collected from traditional histological techniques, which are limited by their smaller viewable area and minimal depth of view. In examining blood vessels, a structure that is $3 \mathrm{D}$ but often analyzed in $2 \mathrm{D}$, we found little standardization of metrics across the field, making comparison to prior methods challenging [13]. By comparing volumetric and vectorized measurements of mouse cerebellum and forebrain, significant differences in vascular density, surface area to volume ratio, and vessel tortuosity were discovered. In addition, each region showed significant differences in quantification of features compared to $2 \mathrm{D}$ images. This suggests that subsampling of tissue may introduce a bias in stereological estimates.

\section{References}

[1] JR Chung et al., Frontiers in Neuroinformatics 5 (2011) 29.

[2] ME Vandenberghe et al., Scientific Reports 6 (2016) 20958.

[3] D Mayerich et al., J Microsc 231(1) (2008) 134-43.

[4] AX Falcão et al., IEEE T Pattern Anal 26(1) (2004) 19-29.

[5] AF Frangi et al., Medical Image Computing and Computer-Assisted Intervention - MICCAI'98 (Vol. 1496), Springer, Berlin Heidelberg, 1998, 130-37.

[6] D Mayerich and J Keyser, IEEE T Vis Comput Gr 15(4) (2009) 670-81.

[7] PS Tsai et al., J Neurosci 29(46) (2009) 14553-70.

[8] K Palágyi and A Kuba, Graph Model Im Proc 61(4) (1999) 199-221.

[9] VNP Vemuri, Feature-Based Analysis of Microvasculature in High Resolution Microscopy Images of Mice Brains, University of Houston, Houston, 2016.

[10] T Wang and I Cheng, Lecture Notes in Computer Science (Vol. 5358 LNCS), Springer, Berlin Heidelberg, 2008, 1051-60.

[11] R Scorcioni et al., Nature Protocols 3(5) (2008) 866-76.

[12] F Cassot et al., Microcirculation 13(1) (2006) 1-18.

[13] WY Leung and MB Jensen, ISRN Neurology (2013) vol 2013:853737.

[14] H Kobayashi et al., Int J Cancer 112(6) (2004) 920-26. 
